\title{
Spermatic Vein
}

National Cancer Institute

\section{Source}

National Cancer Institute. Spermatic Vein. NCI Thesaurus. Code C53050.

The veins arising in the right and left testicles that join to form the spermatic plexus then diverge with the left segment draining into the left renal vein and the right into the inferior vena cava. 